\title{
Azelnidipine, a long-acting calcium channel blocker, could control hypertension without decreasing cerebral blood flow in post-ischemic stroke patients. A ${ }^{123}$ I-IMP SPECT follow-up study
}

\author{
Masaki Watanabe ${ }^{1}$, Teruyuki Hirano ${ }^{1}$, Sadahisa Okamoto $^{1}$, Shinya Shiraishi ${ }^{2}$, Seiji Tomiguchi ${ }^{3}$ \\ and Makoto Uchino ${ }^{1}$
}

Azelnidipine, a long-acting calcium channel blocker, is highly lipid soluble and selective for the vascular wall, and is expected to have an increasing effect on cerebral blood flow (CBF). The aim of this study is to investigate its safety and efficacy in stroke patients in the chronic stage as far as $\mathrm{CBF}$ is concerned using $\mathrm{N}$-isopropyl-p-123/-iodo amphetamine (123/-IMP) single-photon emission computed tomography (SPECT). The patients were orally administered 8 or $16 \mathrm{mg}$ of azelnidipine. Regional CBF was evaluated by ${ }^{123}$ I-IMP SPECT using three-dimensional stereotactic region-of-interest (ROI) template (3D-SRT), a technique using anatomical standardization and ROI template consisting of 636 ROIs for the whole brain. Mean hemispheric CBF was defined as the mean value of the corpus callosum, and the precentral, central, parietal, angular and temporal gyri. Mean hemispheric and regional CBF after 1, 3 and 6 months were analyzed using a one-way repeated-measures analysis of variance. Ten post-ischemic stroke patients with hypertension were enrolled between October 2005 and October 2007, and all of them were well controlled with normal blood pressure (before: $172.3 \pm 16.6 / 88.4 \pm 14.0 \mathrm{~mm} \mathrm{Hg} ; 6$ months: $128.7 \pm 15.9 / 70.9 \pm 10.1 \mathrm{~mm} \mathrm{Hg}$ ). No vascular events were observed during the study period. The mean hemispheric CBF was maintained during the study period (before: $46.0 \pm 9.7 \mathrm{ml}$ per $100 \mathrm{~g}$ per min; 6 months: $49.3 \pm 11.1 \mathrm{ml}$ per $100 \mathrm{~g}$ per min). The regional CBF was also maintained. In the chronic stage of ischemic stroke, azelnidipine could safely decrease systemic blood pressure without decreasing CBF. Hypertension Research (2010) 33, 43-48; doi:10.1038/hr.2009.179; published online 30 October 2009

Keywords: azelnidipine; calcium channel blocker; cerebral blood flow; ischemic infarct

\section{INTRODUCTION}

Calcium channel blockers (CCBs) are widely used for the treatment of hypertension and ischemic heart disease. In addition to their antihypertensive and cardioprotective effects, many trials showed that CCBs decreased the incidence of stroke. ${ }^{1-3}$ However, in cases of cerebral blood flow (CBF) hemodynamic compromise, a reduction in blood pressure (BP) would decrease the $\mathrm{CBF}$ if the pressure decreases below the lower limit of CBF autoregulation. Antihypertensive therapy itself may induce the risk of ischemic brain complications by a decrease in CBF. Many studies have shown that different CCBs have different effects on CBF. Nilvadipine, a medium-acting CCB, increases the regional $\mathrm{CBF}$ and cerebral hemodynamics. ${ }^{4}$ However, other long-acting CCBs such as amlodipine showed no improvement in $\mathrm{CBF}^{5,6}$

Azelnidipine is a new dihydropyridine calcium channel antagonist that is selective for L-type calcium channels and was recently approved in Japan for the treatment of patients with hypertension. ${ }^{7}$ It has antihypertensive effects comparable with amlodipine. ${ }^{8}$ It is also more lipid soluble and has higher selectivity for the vascular wall than older generation CCBs, and the blood flow to the brain was significantly increased in animal models that were treated with azelnidipine. ${ }^{9}$ However, the effect of azelnidipine on the CBF of patients with stroke has not been addressed. The aim of this study is to test its safety and efficacy in hypertensive stroke patients in the chronic stage as far as $\mathrm{CBF}$ is concerned using $N$-isopropyl- $p^{-123} \mathrm{I}$-iodo amphetamine ( ${ }^{123}$ I-IMP) single-photon emission computed tomography (SPECT).

\section{METHODS}

Subjects, inclusion and exclusion criteria

The study was set with a prospective open-label, single-arm design. The study protocol was reviewed and approved by the institutional review board of our hospital, and informed written consent was obtained from all participants. The inclusion criteria were (1) males or females aged over 20 years; (2) more than 4 weeks must have passed since the last ischemic stroke and the neurological

${ }^{1}$ Department of Neurology, Graduate School of Medical Sciences, Kumamoto University, Kumamoto, Japan; ${ }^{2}$ Department of Radiology, Graduate School of Medical Sciences, Kumamoto University, Kumamoto, Japan and ${ }^{3}$ Department of Diagnostic Radiology, School of Health Sciences, Kumamoto University, Kumamoto, Japan 
status must be stable; and (3) systolic BP of $>140 \mathrm{~mm} \mathrm{Hg}$ and/or a diastolic BP of $>90 \mathrm{~mm} \mathrm{Hg}$.

Exclusion criteria were patients taking other CCBs at the time of enrollment; secondary hypertension; severe systemic complications involving the heart, lungs, liver, or kidneys; within 6 months after acute myocardial infarction; poorly controlled diabetes mellitus; intolerance to CCBs; pregnancy; administration of azole antifungal drugs or human immunodeficiency virus protease inhibitors.

Blood pressure was measured, with patients in the sitting position after at least $10 \mathrm{~min}$ at rest, using a sphygmomanometer, by the same examiner on the same arm. Heart rate was also recorded. After enrollment, the patients underwent pretreatment examinations including a chest radiograph, electrocardiography, routine hematological and blood biochemical analysis, and ${ }^{123}$ I-IMP SPECT. The analyzed parameters included red and white blood cell and platelet counts, hemoglobin, hematocrit, glutamic oxaloacetic transaminase, glutamicpyruvic transaminase, $\gamma$-glutamyl transpeptidase, alkaline phosphatase, total bilirubin, $\mathrm{C}$ reactive protein, blood urea nitrogen, serum creatinine, electrolytes (sodium, potassium and chloride), total cholesterol, low-density lipoprotein, high-density lipoprotein, triglycerides and fasting blood sugar.

On the completion of pretreatment examinations, azelnidipine at a dose of 8 or $16 \mathrm{mg}$ daily was prescribed. Other antihypertensive drugs than CCBs could be given if the BP could not be controlled at an optimal range only with azelnidipine.

Blood pressure, routine blood tests and CBF were similarly evaluated after 1 , 3 and 6 months. Patients were asked to visit every $2-4$ weeks so that their neurological status could be examined and BP measured at each visit. Drug compliance was also checked at each visit by the investigator. Vascular events during the study period were classified as ischemic heart disease, for example, angina pectoris and myocardial infarction; and ischemic stroke, including transient ischemic attacks.

\section{Table 1 Demographic characteristics of patients}

\begin{tabular}{|c|c|c|c|c|c|}
\hline Case & $\begin{array}{c}\text { Age } \\
\text { (years) }\end{array}$ & Sex & Diagnosis & Lesion of infarction & $\begin{array}{l}\text { Vascular risk } \\
\text { factors }\end{array}$ \\
\hline 1 & 62 & $M$ & Lacunar & Left basal ganglia & HT and DM \\
\hline 2 & 78 & $M$ & Cardiogenic & Left caudate & HT \\
\hline 3 & 70 & $\mathrm{M}$ & Atherothrombotic & Right CR & $\mathrm{HT}$ and $\mathrm{DL}$ \\
\hline 4 & 72 & $\mathrm{~F}$ & Lacunar & Right CR & $\mathrm{HT}$ and $\mathrm{DL}$ \\
\hline 5 & 79 & $\mathrm{~F}$ & Atherothrombotic & Left caudate & $\mathrm{HT}$ and $\mathrm{DL}$ \\
\hline 6 & 70 & $M$ & Lacunar & Left CR & HT \\
\hline 7 & 68 & $\mathrm{M}$ & Atherothrombotic & Right parietal & $\mathrm{HT}$ and $\mathrm{DL}$ \\
\hline 8 & 73 & $\mathrm{~F}$ & Atherothrombotic & Right DWM & HT \\
\hline 9 & 70 & $\mathrm{~F}$ & Cardiogenic & Left SBZ & HT \\
\hline 10 & 72 & M & Atherothrombotic & Left SCA territory & $\mathrm{HT}$ and DM \\
\hline
\end{tabular}

Abbreviations: $\mathrm{CR}$, corona radiata; DL, dyslipidemia; DM, diabetes mellitus; DWM, deep white matter, F, female; HT, hypertension; M, male; SBZ, superficial border zone, SCA, superior cerebellar artery.
Stroke was classified according to the Trial of ORG 10172 in Acute Stroke Treatment (TOAST) criteria $^{10}$ by comparing clinical symptoms, responsible lesions on magnetic resonance imaging and steno-occlusive lesions on ultrasound analysis, magnetic resonance angiography and cerebral angiography.

Between October 2005 and October 2007, a total of 11 patients who fulfilled the inclusion criteria were included in the study after obtaining their informed consent. Ten patients completed the study but one patient discontinued because of heart failure resulted from a valve disease. Finally, we analyzed 10 patients and 20 hemispheres. The clinical features of these 10 patients are summarized in Table 1 . They were seven males and three females, and their ages ranged from 62 to 79 years (mean \pm s.d.: $71.3 \pm 1.7$ ). The types of ischemic stroke, according to the TOAST classification, were small vessel disease in three, large artery atherosclerosis in five and cardiogenic stroke in two. The time between the last ischemic stroke and the enrollment was an average of 425.8 days (range: 37-2026).

\section{SPECT imaging and analysis}

General procedure. We used a two-head gamma camera (Millennium VG, General Electric Medical Systems, Milwaukee, WI, USA) equipped with a lowenergy general-purpose collimator. We loaded ${ }^{123}$ I-IMP ( $167 \mathrm{MBq}$ in $\left.1.5 \mathrm{ml}\right)$ into an extension tube connected to a $20-\mathrm{ml}$ syringe and injected it as a bolus into a vein in the right or left arm over $10 \mathrm{~s}$ with $20 \mathrm{ml}$ of saline. Planar images of the brain (cerebral radioactivity at $5 \mathrm{~min}$ after injection: $\mathrm{Cb} 5$ ) were obtained for $1 \mathrm{~min}$ $\left(128 \times 128\right.$ matrix) at $5 \mathrm{~min}$ after bolus injection of ${ }^{123}$ I-IMP. Planar images of the brain were also obtained before $\left(\mathrm{Cb}_{\text {pre }}\right)$ and after $\left(\mathrm{Cb}_{\text {post }}\right)$ SPECT imaging. SPECT imaging of the brain was carried out for $20 \mathrm{~min}$ (continuous mode, 60 steps, $20 \mathrm{~s}$ per step, magnification $\times 1.5,128 \times 128$ matrix) at $15 \mathrm{~min}$ after bolus injection of ${ }^{123}$ I-IMP. Axial SPECT images were reconstructed by the filtered back projection method using a Ramp filter. A Butterworth filter with an order of 10 and a cutoff of 0.45 was used as the pre-reconstruction filter. The reconstructed slice thickness was $2.95 \mathrm{~mm}$. Correction for attenuation or scatter was not performed.

Continuous arterial blood sampling. Continuous arterial blood sampling was carried out with a suction pump through an extension tube connected to a 27gauge Teflon catheter in the right radial artery. The total dead space for sampling was about $1 \mathrm{ml}$. Continuous sampling at a constant rate of $1 \mathrm{ml} \mathrm{min}^{-1}$ was started immediately after bolus injection of ${ }^{123}$ I-IMP and stopped $5 \mathrm{~min}$ later. The total radioactivity of the blood sample $(1 \mathrm{ml}$ : whole blood count) was measured by a scintillation well counter immediately after the completion of continuous arterial blood sampling. A mixture of $0.5 \mathrm{ml}$ of blood and $2 \mathrm{ml}$ of octanol was vortexed and then centrifuged at 3000 r.p.m. for $20 \mathrm{~min}$. Then the radioactivity in $1.0 \mathrm{ml}$ of the octanol fraction (octanol count) was measured by a scintillation well counter. Next, the arterial input function for $5 \mathrm{~min}(\mathrm{AIF}(5))$ was obtained by the following equation:

$$
\begin{aligned}
\operatorname{AIF}(5)= & 4 \times(\text { octanol count } / \text { whole blood count }) \\
& \times \text { whole blood count }(\text { count } / \mathrm{ml})
\end{aligned}
$$

Octanol count is obtained by measurement of $1 \mathrm{ml}$ octanol fraction extracted from the mixture of $0.5 \mathrm{ml}$ of whole blood and $2 \mathrm{ml}$ of octanol. Therefore, the
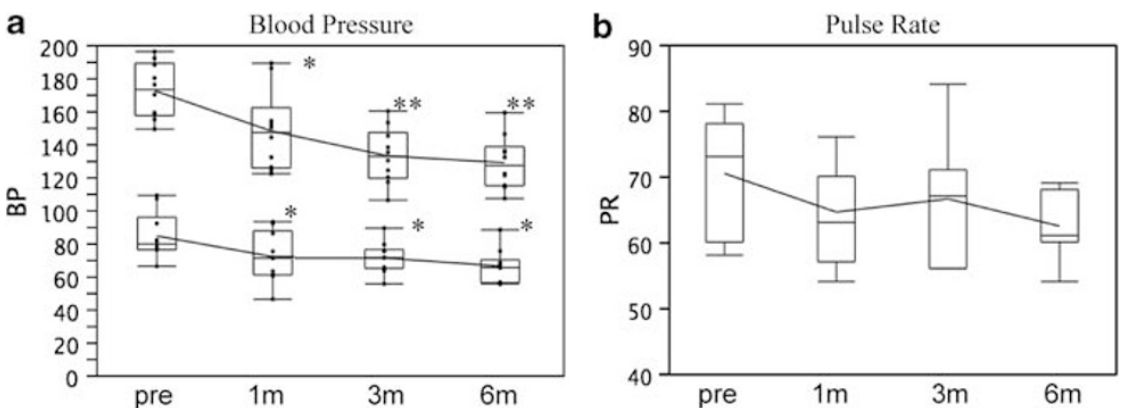

Figure 1 (a) Upper box, serial change of systolic blood pressure. They were all well controlled to optimal systolic blood pressure with statistical significance (paired $t$-test). Lower box, serial change of diastolic blood pressure. They were well controlled to optimal blood pressure with statistical significance (paired $t$-test) $\left({ }^{*} P<0.05\right.$ or $\left.{ }^{* *} P<0.001\right)$. (b) Pulse rate did not change significantly. 
multiplying factor of 4 is necessary for the calculation of octanol fraction of $1 \mathrm{ml}$ whole blood.

\section{Measurement of CBF}

According to the microsphere model, ${ }^{11} \mathrm{rCBF}$ (ml per $100 \mathrm{~g}$ per min) was calculated as follows:

$$
\mathrm{rCBF}=\mathrm{rCb}(5) / \int \mathrm{Ca}(t) \mathrm{d} t=\operatorname{rCb}(5) / \operatorname{AIF}(5)
$$

where $\mathrm{rCb}(5)$ is the regional radioactivity $\left(\mu \mathrm{Cig}^{-1}\right)$ of the brain tissue at $5 \mathrm{~min}$ after injection of ${ }^{123} \mathrm{I}$-IMP, $\mathrm{Ca}(\mathrm{t})$ is the radioactivity $\left(\mu \mathrm{Ci} \mathrm{ml}^{-1}\right)$ vs time profile of ${ }^{123} \mathrm{I}$-IMP in arterial blood, and $\int \mathrm{Ca}(t) \mathrm{d} t(\mathrm{~min} * \mu \mathrm{Ci} / \mathrm{ml})$ is the integral of $\mathrm{AIF}(5)\left(\min \times \mu \mathrm{Ci} \mathrm{ml}^{-1}\right) \cdot \mathrm{rCb}(5)$ was also calculated as follows: $\mathrm{rCb}(5)=$ brain SPECT count $\times\left[\mathrm{Cb}(5)\right.$ count/average of the $\mathrm{Cb}_{\text {pre }}$ and $\mathrm{Cb}_{\text {post }}$ counts $]$. Then, regional $\mathrm{CBF}$ could be determined by equation (1).

\section{Data analysis}

Three-dimensional stereotactic region-of-interest (ROI) template (3D-SRT), a technique using anatomical standardization and a ROI template consisting of 636 ROIs of the entire brain, was introduced to quantitate local CBF. ${ }^{12}$ Mean hemispheric CBF was defined as the mean value of the corpus callosum, and the precentral, central, parietal, angular and temporal gyri, which are all located in the anterior circulation. We analyzed the following factors on SPECT: (1) serial change in the mean hemispheric CBF; (2) differences in serial mean hemispheric $\mathrm{CBF}$ changes between infarcted and non-infarcted hemispheres; (3) serial change of the regional CBF in the corpus callosum, the precentral, central, parietal, angular and temporal gyri, lenticular nucleus, thalamus and hippocampus.

\section{Statistical analysis}

Values are expressed as the mean \pm s.d. in the text, table and figures. BP and heart rate after 1, 3 and 6 months were compared with the baseline data using the paired $t$-tests. The CBF was analyzed using a one-way repeated-measures analysis of variance. A $P$-value $<0.05$ was considered statistically significant.

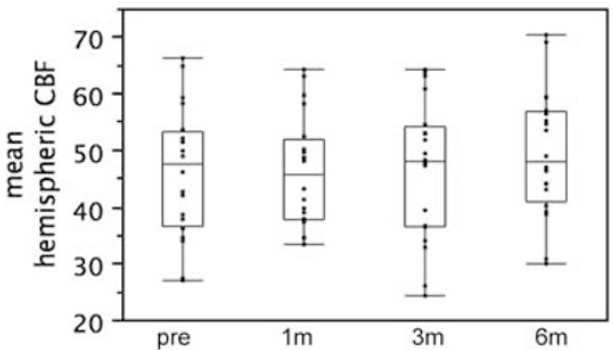

Figure 2 Serial change in mean hemispheric cerebral blood flow (CBF). No significant change was observed (one way repeated-measured analysis of variance (ANOVA), $P=0.74$ ).
Statistical analysis was carried out using statistical analysis software (JMP, version 6.0.3, SAS Institute, Cary, NC, USA).

\section{RESULTS}

Eight of nine patients received azelnidipine $16 \mathrm{mg}$, and the remaining one patient received $8 \mathrm{mg}$. They were all well controlled at an optimal systolic BP with statistical significance (before: $172.3 \pm 16.6 \mathrm{~mm}$ Hg; 1 month: $147.9 \pm 24.0 \mathrm{~mm} \mathrm{Hg} ; 3$ months: $132.8 \pm 16.8 \mathrm{~mm} \mathrm{Hg}$; 6 months: $128.7 \pm 15.9 \mathrm{~mm} \mathrm{Hg}$. Paired $t$-test: $P<0.05$ in 1 month, $P<0.001$ in 3 and 6 months) (Figure 1). Diastolic BP were also well controlled at an optimal level with statistical significance (before: $88.4 \pm 14.0 \mathrm{~mm} \mathrm{Hg} ; 1$ month: $75.8 \pm 15.1 \mathrm{~mm} \mathrm{Hg} ; 3$ months: $75.8 \pm$ $7.5 \mathrm{~mm} \mathrm{Hg} ; 6$ months: $70.9 \pm 10.1 \mathrm{~mm} \mathrm{Hg}$. Paired $t$-test: $P<0.05)$ (Figure 1). Heart rate did not change significantly (before: $70.4 \pm 8.7$ beats per min; 1 month: $64.6 \pm 7.7$ beats per min; 3 months: $66.6 \pm 9.6$ beats per min; 6 months: $62.4 \pm 5.2$ beats per min. Paired $t$-test: $P=0.02$ ) (Figure 1).

Neither abnormal data on blood tests nor vascular events were observed during the study period.

When all hemispheres were summarized and evaluated, the mean hemispheric CBF had not changed significantly (before: $46.0 \pm 9.7 \mathrm{ml}$ per $100 \mathrm{~g}$ per min; 1 month: $46.0 \pm 9.7 \mathrm{ml}$ per $100 \mathrm{~g}$ per min; 3 months: $46.6 \pm 12.0 \mathrm{ml}$ per $100 \mathrm{~g}$ per min; 6 months: $49.3 \pm 11.1 \mathrm{ml}$ per $100 \mathrm{~g}$ per $\mathrm{min}$ ) (one way repeated-measures analysis of variance, $P=0.74$ ) (Figure 2). Furthermore, by a separate analysis of both the infarcted and non-infarcted hemispheres, significant changes in the mean hemispheric CBF were not observed (infracted hemisphere: $n=10$, before: $45.5 \pm 12.3 \mathrm{ml}$ per $100 \mathrm{~g}$ per min; 6 months: $48.6 \pm 11.3 \mathrm{ml}$ per $100 \mathrm{~g}$ per $\mathrm{min}, P=0.92$; non-infarcted hemisphere: $n=10$, before: $46.6 \pm 11.1 \mathrm{ml}$ per $100 \mathrm{~g}$ per min; 6 months: $50.1 \pm$ $11.4 \mathrm{ml}$ per $100 \mathrm{~g}$ per min, $P=0.87$ ) (Figure 3 ). The regional CBF did not show any significant change in all regions (Table 2). Case 1 was shown in Figure 4.

\section{DISCUSSION}

To the best of our knowledge, this is the first study that shows that azelnidipine can safely decrease the systemic BP without decreasing CBF in the chronic stage of ischemic stroke. There are three points in this study. First, mean hemispheric CBF was maintained for 6 months under administration of the drug, although the systemic BP was significantly lowered. Second, hemispheric CBF was maintained regardless of the presence of infracted and/or peri-infarcted tissues. Finally, regional CBF was also maintained for 6 months.

The antihypertensive effect of azelnidipine is consistent with some previous mega studies using amlodipine, ${ }^{1-3}$ indicating that the antihypertensive effect continues for several months to years of drug
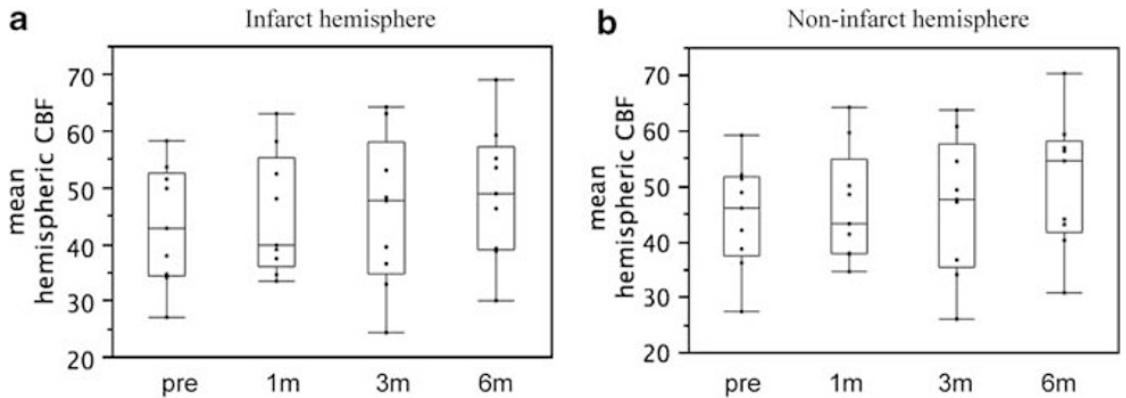

Figure 3 (a) Infarct hemisphere $(n=10)$. Separate analysis for affected hemispheres showed no significant change in cerebral blood flow (CBF; $P=0.92)$. (b) Non-infarct hemisphere $(n=10)$. Separate analysis for non-infarct hemisphere showed no significant change in CBF $(P=0.87)$. 
Table 2 Serial change of the regional CBF

Regional CBF (mean \pm s.d., $\mathrm{ml}$ per $100 \mathrm{~g}$ per min)

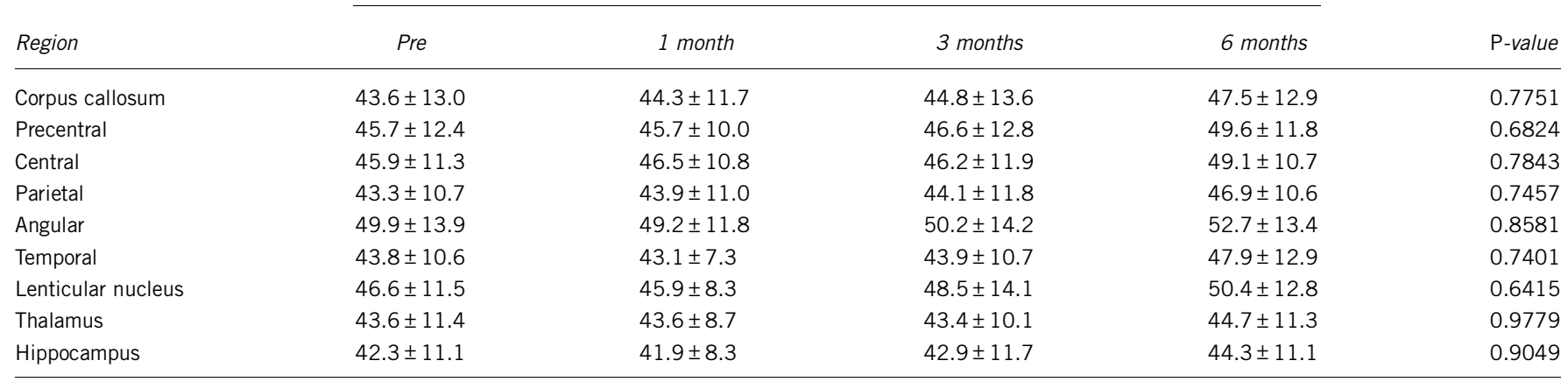

Abbreviation: $\mathrm{CBF}$, cerebral blood flow.
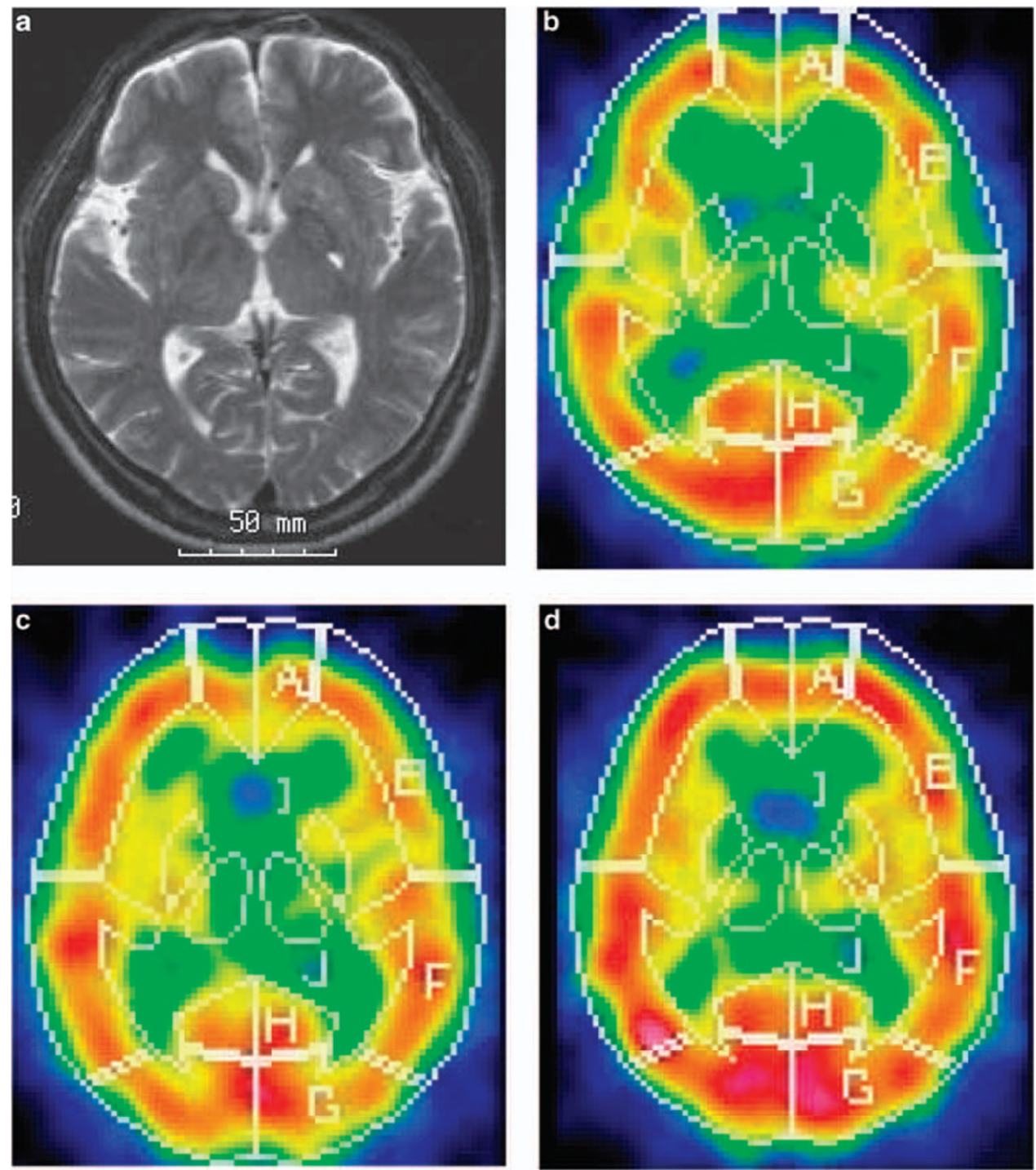

Figure 4 Case 1 (62-year-old male) suffered from lacunar infarction in left basal ganglia. (a) Magnetic resonance imaging (MRI) T2-weighted images (T2WI) and (b) $\mathrm{N}$-isopropyl-p-123/-iodo amphetamine (123/-IMP) single-photon emission computed tomography (IMP SPECT) on the level of the basal ganglia at pretreatment. (c) Three months after treatment with $16 \mathrm{mg}$ of azelnidipine and (d) 6 months after. Significant CBF increase was seen in bilateral hemispheres. 
administration. BP depression in the subacute stage of ischemic stroke, that is, the natural course, did not seem to be suitable in this study because all the patients were enrolled in the chronic stage at 1 month after an ischemic attack (median: 426 days). Furthermore, the systolic BP gradually decreased to the target BP range described in the VALUE study ${ }^{3}$ from 3 to 6 months, although the pretreatment BP was higher than in other studies because of the post-ischemic stroke status. We considered that BP was lowered by the effect of azelnidipine, and not by the natural course post-ischemic event.

Activation of the sympathetic nervous system and the increase in heart rate by short-acting CCBs were important issue. However, the increase in heart rate was not observed in this study. Azelnidipine has the characteristic of not increasing the heart rate in stroke-prone spontaneously hypertensive rats. ${ }^{13}$ The inhibition of sympathetic tone, in addition to the direct effect on the sinus node is presumed to be the main mechanism. Our present clinical data thus support previous experimental findings.

Many studies have shown that CCBs have some effects on CBF. Nilvadipine, a medium-acting $\mathrm{CCB}$, increases the regional $\mathrm{CBF}$ and improves the cerebral hemodynamics. ${ }^{4}$ On the other hand, $\mathrm{CBF}$ autoregulation is impaired by some classical CCBs, such as nimodipine and nitrendipine. ${ }^{14-16}$ Some reports showed that other longacting CCBs such as amlodipine showed no improvement on CBF in humans, ${ }^{5,6}$ and others showed that amlodipine ${ }^{17}$ and benidipine ${ }^{18}$ improve the lower limit of CBF autoregulation in animal models. Although there have not been any reports on the effect of azelnidipine on the $\mathrm{CBF}$ in stroke patients, our results postulated that azelnidipine does not have a negative effect on $\mathrm{CBF}$.

Several mechanisms of action for CBF after treatment with azelnidipine are speculated. First, a primary vasodilatation effect by L-type voltage-dependent calcium channels blockade was considered. Similar to other long-lasting dihydropyridine CCBs such as amlodipine ${ }^{17}$ and benidipine, ${ }^{18}$ it may also shift the lower limits of CBF autoregulation in the direction of a lower BP by changing the cerebral resistance of vessels. However, we did not evaluate the hemodynamics by using acetazolamide, so it is uncertain if azelnidipine can actually shift the lower limits of CBF. Second, Kimura Y et al. ${ }^{19}$ reported that antihypertensive treatment with azelnidipine enhanced the endothelial nitric oxide synthase expression levels in the brain. Endothelialderived nitric oxide has crucial roles in the vascular physiological action. ${ }^{20}$ Nitric oxide is a vasodilator and exerts anti-inflammatory, anti-thrombotic, anti-apoptotic and anti-proliferative actions. Therefore, azelnidipine may protect the cerebral circulation by increasing endothelial NO production. The antioxidative property that azelnidipine was reported to have ${ }^{21}$ may be involved in the normalization of the dysautoregulation of cerebral circulation. Third, sympathetic nervous activity, which increases during hypertension, not only contributes to the adjustment to circulatory stress but also to a sustained increase in vascular resistance and arterial pressure. ${ }^{22-24}$ Some CCBs induce an increase in plasma catecholamine, which is involved in the sympathetic nervous system. ${ }^{25}$ In stroke-prone spontaneously hypertensive rats, oral administration of azelnidipine (20 $\mathrm{mg} \mathrm{kg}^{-1}$ per day) did not affect urinary norepinephrine excretion, whereas other antihypertensive drugs significantly elevated it despite equal levels of antihypertensive doses, ${ }^{19}$ suggesting that antihypertensive treatment with azelnidipine attenuates reflex-induced sympathetic activation and reduces vascular resistance. Finally, azelnidipine has more lipid solubility and selectivity for the vascular wall than older generation CCBs. ${ }^{9,26}$ It is retained in the vascular wall after clearance from the blood, and therefore has a relatively longer effect on the cerebral vessels. The above possible beneficial properties are not necessarily shared by all CCBs; hence, it is not a class effect, but may be drug-specific effects of azelnidipine.

This is the first report of evaluating CBF by 3D-SRT in patients with stroke who are treated with CCBs. In previous reports, the CBF study was evaluated by other methods than 3D-SRT. So long as the ROIs were manually selected, the obtained results fluctuated considerably with subtle changes in their positioning, and it was possible to overlook important information in an area where ROI was not set. 3D-SRT is a fully automated regional CBF quantification program and compensates for the weakness of manual quantitative methods and allows us to use universal ROIs, thus excluding subjectivity. ${ }^{12}$ With its improved objectivity and excellent reproducibility, this 3D-SRT method may detect solid results in this study.

There are several limitations to this study. First, this is an openlabel, single-arm, uncontrolled study. Owing to the ethical perspectives concerning non-antihypertensive therapy in post-ischemic stroke patients with hypertension, we were unable to create a control group. Second, the number of enrolled patients is too small because SPECT is too expensive to recruit a large number of patients. Third, regarding the measurement of $\mathrm{BP}$, ambulatory $\mathrm{BP}$ measurement was not introduced in this study. It prevents the influence of white coat hypertension during normal activities and can assess BP in the nighttime and early-morning phases. Finally, $\mathrm{PaCO}_{2}$ levels that could influence on CBF were not measured during arterial blood samplings. Because our patients did not have respiratory diseases and have normal respiration during the test, we estimated that $\mathrm{PaCO}_{2}$ levels were in normal range and did not measure them. Despite such limitations of this study, our results suggest that azelnidipine may have a beneficial effect on serial CBF in hypertensive patients with ischemic stroke.

In conclusion, in the chronic stage of ischemic stroke, azelnidipine can safely decrease systemic BP without decreasing mean hemispheric and regional CBF. These results suggest that azelnidipine may be therapeutically useful for the treatment of hypertensive patients with post-ischemic stroke.

\section{CONFLICT OF INTEREST}

The authors declare no conflict of interest.

1 The Syst-Eur Investigators. Prevention of dementia in randomized double-blind placebo-controlled Systolic Hypertension in Europe (Syst-Eur) trial. Lancet 1998; 352 1347-1351.

2 ALLHAT Officers and Coordinators for ALLHAT Collaborative Research Group. Major Outcomes in High-Risk Hypertensive Patients Randomized to Angiotensin-Converting Enzyme Inhibitor or Calcium Channel Blocker vs Diuretic. The Antihypertensive and Lipid-Lowering Treatment to Prevent Heart Attack Trial (ALLHAT). JAMA 2002; 288: 2981-2997.

3 Julius S, Kjeldsen SE, Weber M, Brunner HR, Ekman S, Hansson L, Hua T, Laragh J, McInnes GT, Mitchell L, Plat F, Schork A, Smith B, Zanchetti A. Outcomes in hypertensive patients at high cardiovascular risk treated with regimens based on valsartan or amlodipine: the VALUE randomized trial. Lancet 2004; 363: 2022-2031.

4 Ogasawara K, Noda A, Yasuda S, Kobayashi M, Yukawa H, Ogawa A. Effect calcium antagonist on cerebral blood flow and oxygen metabolism in patients with hypertension and chronic major cerebral artery occlusion: a positron emission tomography study. Nucl Med Commnun 2003; 24: 71-76.

5 Pandita-Gunawardena ND, Clarke EM. Amlodipine lowers blood pressure without affecting cerebral blood flow as measured by single photon emission computed tomography in elderly hypertensive subjects. Age Ageing 1999; 28: 451-457.

6 Shimizu H, Tominaga T, Fujiwara S. Amlodipine lowers blood pressure without significant effect on cerebral blood flow in hypertensive patients with a history of stroke: a quantitative single photon emission computed tomography study. J Stroke Cerebrovasc Dis 2003; 12: 266-270.

7 Wellington K, Scott LJ. Azelnidipine. Drugs 2003; 4: 122-125.

8 Kuramoto K, Ichikawa S, Hirai A, Kanada S, Nakachi T, Ogihara T. Azelnidipine and amlodipine: a comparison of their pharmacokinetics and effects on ambulatory blood pressure. Hypertens Res 2003; 26: 201-208. 
9 Yagil Y, Lustig A. Azelnidipine (CS-905), a novel dihydropyridine calcium channel blocker with gradual onset and prolonged duration of action. Cardiovasc Drug Rev 1995; 13: 137-148.

10 The publications Committee for the Trial of ORG 10172 in Acute Stroke Treatment (TOAST) investigators. Low molecular weight heparinoid Org 10172(danaparoid), and outcome after acute ischemic stroke. A randomized, controlled trial. JAMA 1998; 279: 1265-1272.

11 Kuhl DE, Barrio JR, Huang SC, Selin C, Ackermann RF, Lear JL, Wu JL, Lin TH, Phelps ME. Quantifying local cerebral blood flow by $N$-isopropyl- $p$-[123I]iodoamphetamine (IMP) tomography. J Nucl Med 1982; 23: 196-203.

12 Takeuchi R, Matsuda H, Yoshioka K, Yonekura Y. Cerebral blood flow SPET in transient global amnesia with automated ROI analysis by 3DSRT. Eur J Nucl Med Mol Imaging 2004; 31: 578-589.

13 Yamamoto E, Lai ZF, Yamashita T, Tanaka T, Kataoka K, Tokutomi Y, Ito T, Ogawa H, Kim-Mitsuyama S. Enhancement of cardiac oxidative stress by tachycardia and its critical role in cardiac hypertrophy and fibrosis. J Hypertens 2006; 24: 2057-2069.

14 Harris RJ, Branston NM, Symon L, Bayhan M, Watson A. The effects of a calcium antagonist, nimodipine, upon physiological response of the cerebral vasculature and its possible influence upon focal cerebral ischaemia. Stroke 1982; 13: 759-766.

15 Hollerhage HG, Gaab MR, Zumkeller M, Walter GF. The influence of nimodipine on cerebral blood flow autoregulation and blood-brain barrier. J Neurosurg 1988; 69: 919-922.

16 Aihara K. Effects of nitrendipine on the autoregulation of regional cerebral blood flow in the pithed rabbits. Naunyn Schmiedebergs Arch Pharmacol 1989; 339: 469-473.
17 Cai H, Yao H, Ibayashi S, Takaba H, Fujishima M. Amlodipine, a Ca channel antagonist, modifies cerebral blood flow autoregulation in hypertensive rats. Eur J Pharmacol 1996; 313: 103-106.

18 Ikeda J, Yao K, Matsubara M. Effects of benidipine, a long-lasting dihydropyridine$\mathrm{Ca} 2+$ channel blocker, on cerebral blood flow autoregulation in spontaneously hypertensive rats. Biol Pharm Bull 2006; 29: 2222-2225.

19 Kimura Y, Hirooka Y, Sagara Y, Sunagawa K. Long-acting calcium channel blocker, azelnidipine, increases endothelial nitric oxide synthase in the brain and inhibits sympathetic nerve activity. Clin Exp Hypertens 2007; 29: 13-21.

20 Faraci FM, Heistad DD. Regulation of the cerebral circulation: role of endothelium and potassium channels. Physiol Rev 1998; 78: 53-97.

21 Lukic-Panin V, Kamiya T, Zhang HZ, Hayashi T, Tsuchiya A, Sehara Y, Deguchi K, Yamashita T, Abe K. Prevention of neuronal damage by calcium channel blockers with antioxidative effects after transient focal ischemia in rats. Brain Res 2007; 1176: $143-150$.

22 Strandgaard S, Paulson OB. Cerebral autoregulation. Stroke 1984; 15: 413-416.

23 Paulson OB, Waldemar G, Schmidt JF, Strangaard S. Cerebral circulation under normal and pathologic conditions. Am J Cardiol 1989; 63: 2C-5C.

24 Choi JY, Morris JC, Hsu CY. Aging and cerebrovascular disease. Neurol Clin 1998; 16: 687-711.

25 Grossman E, Messerli FH. Effect of calcium antagonists on plasma norepinephrine levels, heart rate, and blood pressure. Am J Cardiol 1997; 80: 1453-1458.

26 Oizumi K, Nishio H, Koike H, Sada T, Miyamoto M, Kimura T. Antihypertensive effects of CS-905, a novel dihydropyridine Ca++ channel blocker. Jpn J Pharmacol 1989; 51: 57-64. 